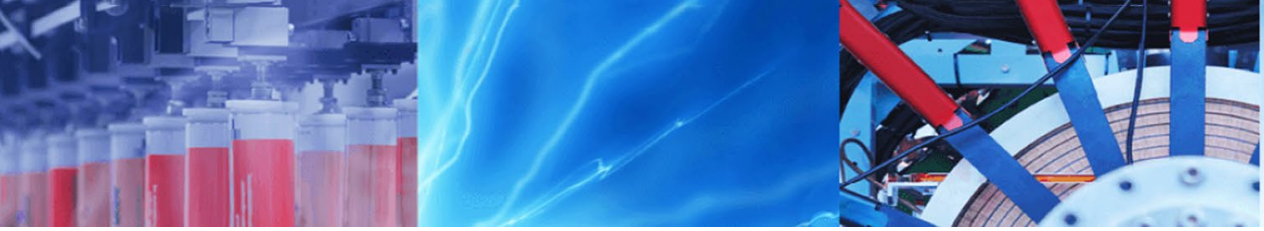

Research Article

\title{
Simultaneous separation and detection chiral fenobucarb enantiomers using UPLC-MS/MS
}

\author{
Weitong Xia ${ }^{1}$ Zongzhe $\mathrm{He}^{1} \cdot \mathrm{Kunming} \mathrm{Hu}^{1} \cdot$ Beibei Gao ${ }^{1}$ Zhaoxian Zhang ${ }^{1} \cdot$ Minghua Wang $^{1} \mathbb{D} \cdot$ Qiang Wang $^{1,2}$
}

(c) Springer Nature Switzerland AG 2019

\begin{abstract}
A reliable and sensitive method was developed and validated for the determination of chiral pesticide fenobucarb enantiomers with UPLC-MS/MS. The fenobucarb enantiomers were baseline separated on Daicel IG-3 chiral column using a mixture of acetonitrile and $0.1 \%$ formic acid water $(60: 40, \mathrm{v} / \mathrm{v})$ as mobile phase. The absolute configuration of fenobucarb enantiomers was confirmed as (R)-(+)-fenobucarb and (S)-(-)-fenobucarb by experimental and calculated electronic circular dichroism spectra. Good linearity $\left(R^{2}>0.9962\right)$ was obtained for the four matrix calibration curves within the range of $0.5-50 \mu \mathrm{g} \mathrm{mL}^{-1}$. The limit of detection of the two enantiomers were in the range of $0.07-0.16 \mu \mathrm{g} \mathrm{L}^{-1}$. The mean recoveries of the two enantiomers ranged from 80.4 to $102 \%$ with intra-day relative standard deviations (RSDs) from 1.8 to $6.6 \%$ and inter-day RSDs from 3.0 to $5.7 \%$ in four matrices. The (R)-(+)-fenobucarb degraded faster than (S)-(-)-isomer in cucumber. The established method can provide additional information for the further research about the enantioselective environmental behaviors of fenobucarb.
\end{abstract}

Keywords Fenobucarb enantiomers · Enantioseparation · Absolute configuration · Stereoselective degradation

\section{Introduction}

Fenobucarb, (R, S)-2-(1-methylpropyl) phenol methylcarbamate (Fig. 1), as a typical carbamate insecticide, was used to control a range of biting and sucking insects in rice and other crops. It has contact action, stomach poisoning, fumigating and some ovicidal action [1]. It could effectively control leaf hoppers, leaf rollers, thrips, stem borers in paddy fields through inhibiting the activity of acetylcholine esterase [2-4]. It is also widely used as a main ingredient of mosquito coil [5]. As a widely used compound, with frequent exposure to body and the environment, the accurate monitoring of fenobucarb is critical for the environmental and ecological risk assessment.

Several methods were applied to detect fenobucarb based on racemate before $[6,7]$. However, it is necessary to establish a chiral analytical method for further studying the environmental fate of fenobucarb enantiomers. The stereostructure of fenobucarb contains a chiral center, which leads to a pair of enantiomers. Optical isomers exhibit similar chemical and physical properties, while their bioactivity, toxicity, absorption, transformation and degradation behaviors are generally enantioselective [8-11]. A research shows that another carbamate chiral pesticide isoprocarb have enantioselective behavior that the more efficient S-isoprocarb degraded faster than that of R-isoprocarb in the cucumber, tomato, cabbage and soil. $[12,13]$. In brief, the different environmental behaviors between the two enantiomers may lead to inaccurate environmental risk assessment.

In this study, a sensitive analytical method was developed for the simultaneous determination of fenobucarb

$\triangle$ Minghua Wang, wangmha@njau.edu.cn; $\bowtie$ Qiang Wang, qiangwang2003@sina.com | ${ }^{1}$ Department of Pesticide Science, College of Plant Protection, Nanjing Agricultural University, State and Local Joint Engineering Research Center of Green Pesticide Invention and Application, Nanjing 210095, People's Republic of China. ${ }^{2}$ Institute of Quality and Standard for Agro-products, Zhejiang Academy of Agricultural Sciences, Hangzhou 310021, People's Republic of China.

SN Applied Sciences (2019) 1:795 | https://doi.org/10.1007/s42452-019-0822-8

Received: 30 March 2019 / Accepted: 21 June 2019 / Published online: 27 June 2019 


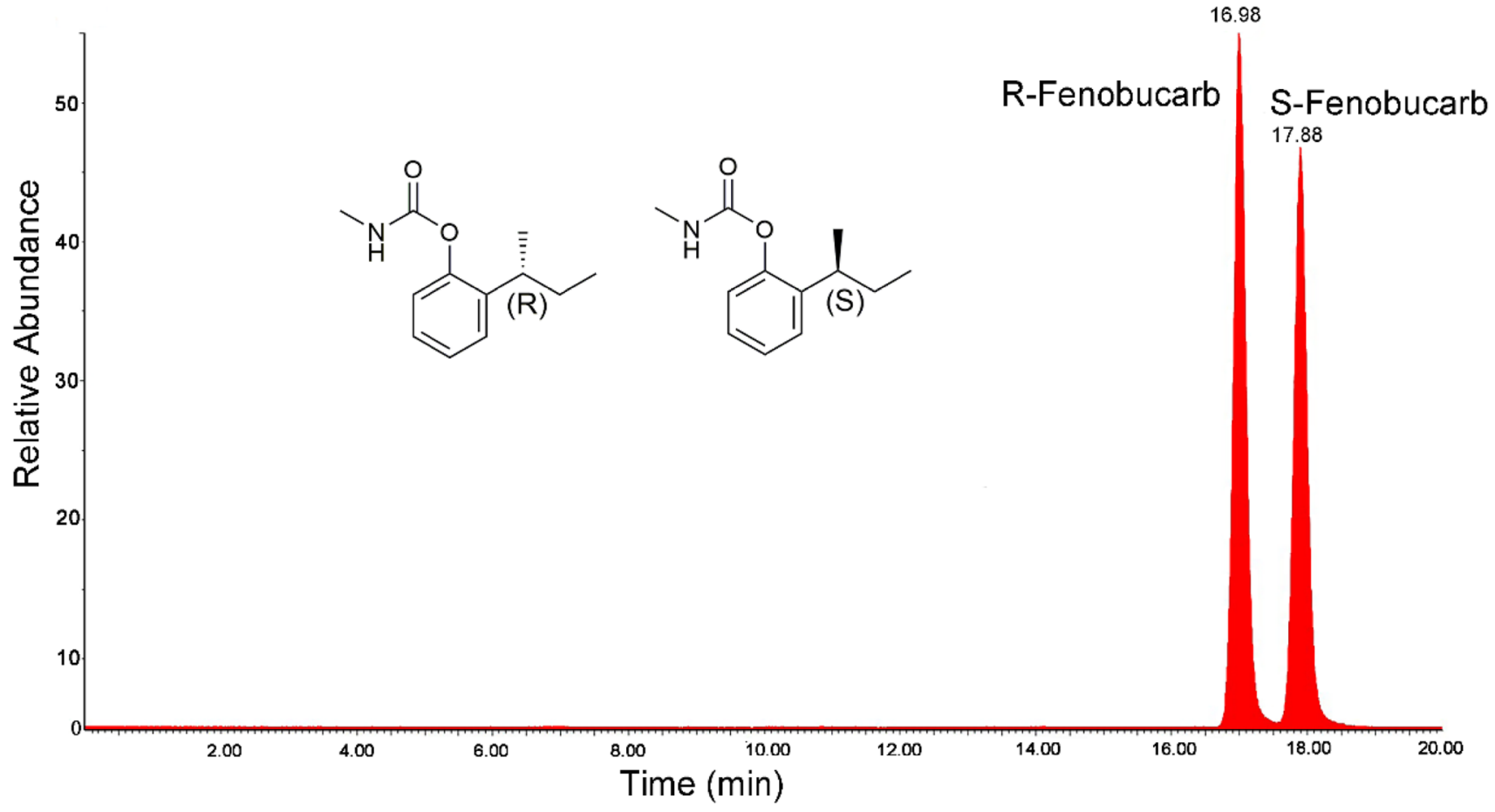

Fig. 1 Chemical structure and the chromatogram of fenobucarb enantiomers

enantiomers in vegetables, crops, soils and water by UPLC-MS/MS using a Daicel IG-3 chiral column. The mobile phase systems were optimized to achieve satisfactory resolution. The absolute configuration of fenobucarb enantiomers was first confirmed by comparing experimental and predicted electronic circular dichroism (ECD) spectra. The optical rotation of enantiomers was measured. The established method was also applied to investigate the enantioselective degradation in cucumber. These results may be helpful for studying the risk assessment of fenobucarb.

\section{Materials and methods}

\subsection{Materials}

The rac-fenobucarb standard ( $\geq 97.0 \%$ purity) was obtained from Jianglai Biological Technology Co., Ltd. (Shanghai, China). Enantiomers of fenobucarb (98.0\% purity) were prepared from Daicel Chiral Technologies Co., Ltd. (Shanghai, China). HPLC-grade acetonitrile was purchased from Merck (Darmstadt, German). Purified water was obtained from MUL-9000 water system (Nanjing Zongxing Water Equipment Co. Ltd, China). All other chemicals were analytical grade and obtained from commercial sources. The rice and cucumbers were bought from local market and the soils and water were taken from the local. All of samples were detected without fenobucarb.
The standards stock solute $\left(1000 \mu \mathrm{g} \mathrm{mL}^{-1}\right)$ of the rac-fenobucarb and enantiomers were dissolved in acetonitrile and stored in $-20^{\circ} \mathrm{C}$. A series of concentration standard working solutions of fenobucarb enantiomer $\left(0.0005-2.5 \mu \mathrm{g} \mathrm{mL}^{-1}\right)$ were prepared in acetonitrile/0.1\% formic acid water $(60: 40, v / v)$ by serial dilution. The $20,000 \mu \mathrm{g} \mathrm{mL}^{-1}$ standard solute of each enantiomer was used to obtain the ECD spectra.

\subsection{Determination of absolute configurations and optical rotation}

The absolute configuration of fenobucarb enantiomers was measured by ECD spectroscopy and calculations. The ECD spectra was got using a Jasco $J 815$ circular dichroism spectropolarimeter (Tokyo, Japan) at $25^{\circ} \mathrm{C}$. The scan wavelength ranged from 200 to $400 \mathrm{~nm}$ at a speed of $50 \mathrm{~nm} \mathrm{~min}{ }^{-1}$. A quartz cell with a path length of $1 \mathrm{~cm}$ was put to use for the scanning and the average of three scans was selected.

The molecular geometries of the fenobucarb enantiomers in their electronic ground state were firstly optimized by Gaussian 09 software using density functional theory (DFT). Afterwards, relying on the time-dependent density functional theory (TDDFT), a reliable molecular orbital information of electronic excitation was obtained. The ECD spectra of the fenobucarb enantiomers was applied by the TDDFT method at a level of B3LYP/6-31+G* in acetonitrile 
with a polarizable continuum model (PCM) solvent model. Both the experimental ECD and predicted ECD were drawn by Origin software (version 8.61) $[14,15]$.

The specific optical rotation of fenobucarb enantiomers was measured by Autopol IV Polarimeter (Rudolph Technologies Co., Ltd., MA, USA) within the light of tungsten halogen lamp at $20^{\circ} \mathrm{C}$ and $589 \mathrm{~nm}$ in triplicate.

\subsection{LC/MS methodology}

Enantiomers were separated and determined on Ultra Performance Liquid Chromatography Tandem Mass Spectrometry (UPLC-MS/MS) comprising a Waters Acquity UPLC coupled with Waters triple-quadrupole mass spectrometer (XEVOTQ-S micro) equipped with an electrospray ionization source (ESI) (Waters, MA, USA). MS/MS analysis was employed under a positive ESI source, with the source temperature of $500{ }^{\circ} \mathrm{C}$, capillary voltage of $2700 \mathrm{~V}$, cone voltage of $86 \mathrm{~V}$, and collision energy of $20 \mathrm{~V}$. The precursor ion of fenobucarb was chosen as $\mathrm{m} / \mathrm{z} 208$; its product quantitative ion was chosen as $\mathrm{m} / \mathrm{z} 152$ with collision voltage of $11 \mathrm{~V}$ and its qualitative ion was $\mathrm{m} / \mathrm{z} 95$ with collision voltage of $17 \mathrm{~V}$ [6]. MS analysis was performed in multiple reaction monitoring (MRM) mode, measuring the fragmentation of fenobucarb. Data acquisition was performed using Mass Lynx 4.2 (Waters, USA) software.

IG-3 column (amylose tris (3-chloro-4-methylphenylcarbamate)) $(250 \times 4.6 \mathrm{~mm}, 3.0 \mu \mathrm{m}$, Daicel Chiral Technologies Co., Ltd, Shanghai, China) was selected as the chiral chromatographic column. The composition of the mobile phase and temperature have a strong influence on the signal response, peak shapes, and retention time. The enantioseparation of fenobucarb was optimized using a mixture of acetonitrile and $0.1 \%$ formic acid water as the mobile phase in ratios of $80: 20$ to $40: 60(\mathrm{v} / \mathrm{v})$ at $20-40{ }^{\circ} \mathrm{C}$. Optimization of chromatographic parameters was evaluated the retention time $(t)$, capacity factor $(k)$, separation factor $(a)$ and resolution factor $(R s)$. In addition, the isolation temperature $\left(\mathrm{T}_{\text {iso }}\right)$, the standard changes in enthalpy $\left(\Delta H^{0}\right)$ and entropy $\left(\Delta S^{0}\right)$ between the enantiomers were used to calculate Van't Holf equations. They were calculated by the following equations

$$
\begin{aligned}
& k=\left(t_{R}-t_{0}\right) / t_{o} \\
& \alpha=k_{2} / k_{1} \\
& R_{s}=2\left(t_{2}-t_{1}\right) /\left(w_{1}+w_{2}\right) \\
& \mathrm{RT} \ln k=-\Delta G^{0}=-\left(\Delta H^{0}-T \Delta S^{0}\right) \\
& \ln \alpha=\ln \left(k_{2}^{\prime} / k_{1}^{\prime}\right)=-\Delta \Delta G^{0} / R T=-\Delta \Delta H^{0} / R T+\Delta \Delta S^{0} / R
\end{aligned}
$$

$t_{R}$ is the retention time of target compound and $t_{0}$ is the void time, $k_{1}$ and $k_{2}$ are the capacity factors for the first and second peak, respectively, and $w_{1}$ and $w_{2}$ are the corresponding peak widths. $R$ is the ideal gas constant and $T$ is the absolute temperature. $\Delta H^{0}$ and $\Delta S^{0}$ are the standard transfer enthalpy and entropy; $\Delta G^{0}$ is the differences in the free energy of the solute in the two phases. $\Delta \Delta G^{0}, \Delta \Delta H^{0}$ and $\Delta \Delta S^{0}$ are the free energy, the enthalpy and the entropy difference between the enantiomers $[16,17]$.

\subsection{Extraction and purification of samples}

For the soil, cucumber, and rice samples, exactly $5.0 \mathrm{~g}$ of minced samples were placed into a $100 \mathrm{~mL}$ polypropylene centrifuge tube. Then, $5 \mathrm{~mL}$ distilled water and $30 \mathrm{~mL}$ acetonitrile were added. After vortex-mixed for $4 \mathrm{~min}$ the tube was ultrasonicated for $10 \mathrm{~min}$. Subsequently, $2.0 \mathrm{~g}$ sodium chloride $(\mathrm{NaCl})$ and $4.0 \mathrm{~g}$ anhydrous magnesium sulfate $\left(\mathrm{MgSO}_{4}\right)$ were added, then the tube was vigorously vortexed for $1 \mathrm{~min}$ followed by centrifuging for $5 \mathrm{~min}$ at $4000 \mathrm{rpm}$. Acetonitrile supernatant $(15 \mathrm{~mL})$ was transferred into a flask and was evaporated to dryness at $45^{\circ} \mathrm{C}$. The extract was redissolved in $2.0 \mathrm{~mL}$ of a mixture of acetonitrile/ $0.1 \%$ formic acid water $(60: 40, \mathrm{v} / \mathrm{v})$, filtered through a $0.22 \mu \mathrm{m}$ nylon syringe filter and diluted 12.5 times for LC-MS/MS analysis.

For the water sample, exactly $5.0 \mathrm{~g}$ were placed into a $150 \mathrm{~mL}$ polypropylene centrifuge tube. Then, $30 \mathrm{~mL}$ ethyl acetate were added. After vortex-mixed for $4 \mathrm{~min}$ the tube was ultrasonicated for $10 \mathrm{~min}$. Subsequently, $2.0 \mathrm{~g} \mathrm{NaCl}$ and $4.0 \mathrm{~g} \mathrm{MgSO}_{4}$ were added, then the tube was vigorously vortexed for $1 \mathrm{~min}$ followed by centrifuging for $5 \mathrm{~min}$ at $4000 \mathrm{rpm}$. Ethyl acetate supernatant $(15 \mathrm{~mL})$ was transferred into a flask and was evaporated to dryness at $45^{\circ} \mathrm{C}$. The extract was redissolved in $2.0 \mathrm{~mL}$ of a mixture of acetonitrile $/ 0.1 \%$ formic acid water $(60: 40, \mathrm{v} / \mathrm{v})$, filtered through a $0.22 \mu \mathrm{m}$ nylon syringe filter and diluted 12.5 times for LC-MS/MS analysis.

\subsection{Method validation}

Matrix effect, linearity, limit of detection (LOD), limit of quantification (LOQ), accuracy, intra- and inter-day accuracy, precision, stability as well as specificity are important parameters of the detection method validation [18].

The series concentration of the standard solutions of fenobucarb enantiomers were prepared in solvent and matrix-matched solutions at the range of $0.5-50 \mu \mathrm{g} \mathrm{L}^{-1}$. The linearity was determined by linear regression analysis of both standard solution and matrix-matched calibration curves. The matrix effects were displayed on the slope ratio of the matrix-matched standard calibration curves and solvent calibration curves. Generally, there is no matrix 
effect when the slope ratio between 0.9 and 1.1, but the slope ratios were out of range, there were matrix-induced enhancement or suppression effects. The stability of fenobucarb enantiomers in the solvent and the matrices was determined for 6 months. Student's $t$ test $(P<0.05)$ was used to evaluate the results of the stability test.

The matrix-dependent LODs and LOQs for both enantiomers are defined to be the concentration that produced a signal-to-noise $(\mathrm{S} / \mathrm{N})$ ratio of 3 and 10 by analyzing spiked with fenobucarb enantiomers [19].

The accuracy and precision were evaluated by recovery experiments in the four matrices (water, soil, cucumber, and rice) at three spiked concentrations $\left(5,50\right.$ and $\left.500 \mu \mathrm{g} \mathrm{L}^{-1}\right)$ in quintuplicate over the course of 3 days. The relative standard deviation (RSD) for the enantiomers were calculated by intraand inter-day assays. The mean recoveries and the intra-day and inter-day RSDs were used to evaluate the accuracy and precision.

\subsection{Field experiment}

A cucumber field was divided into four blocks $\left(30 \mathrm{~m}^{2}\right)$ isolated by a buffer zone (Nanjing, China). the dissipation and the control were designed with three replicate plots. The $50 \%$ fenobucarb emulsifiable water solution was sprayed at a dosage of $562 \mathrm{~g}$ a.i. $\mathrm{ha}^{-1}$. The representative cucumber samples were collected from each plot at $2 h, 1,3,5,7,10$, $14 \mathrm{~d}$ after spraying. All the samples were homogenized and stored at the $-20^{\circ} \mathrm{C}$. The enantioselective degradation of fenobucarb enantiomers in cucumbers were represented by enantiomer fraction (EF) [20].

$\mathrm{EF}=[$ concentration of $R /$ concentration of $(S+R)]$

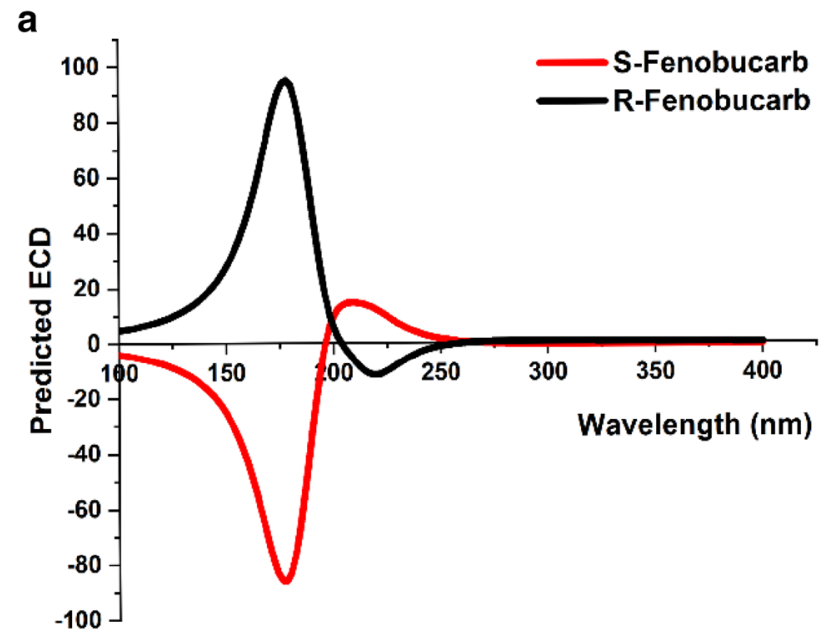

The EF values below 0.5 shows $\mathrm{R}$-fenobucarb degrades preferentially and the EF values over 0.5 shows opposite [21].

\section{Results and discussion}

\subsection{The specific optical rotation and absolute configuration}

The specific optical rotation of peak 1 was measured as $[\alpha]_{D}^{20}=+10.5^{\circ}(c=1.0$, acetontrile), and peak 2 as $[\alpha]_{D}^{20}=-10.6^{\circ}$ ( $c=1.0$, acetontrile). The absolute configurations of the enantiomers were measured using a combination of experimented and predicted ECD spectrum. The predicted ECD spectra of lowest energy conformers are shown in Fig. 2a. And the two experimental circular dichroism (CD) curves were nearly mirror images (Fig. 2b). The predicted ECD spectra were in good agreement with the available experimental spectra. The results confirmed that the first eluted enantiomer (peak 1) was (R)-(+)fenobucarb and the second eluted enantiomer (peak 2) was (S)-(-)-fenobucarb.

\subsection{Optimization of chromatographic condition}

To increase response value on the instrument under positive ESI source, the water was replaced by $0.1 \%$ formic acid water as the half of mobile phase. The effects of the mobile phase and temperature on chiral separation were shown in Table 1. The separation factor and resolution factor were increased and retention time of the enantiomers was extended as the proportion of water increased [22]. Although the best resolution was obtained while the

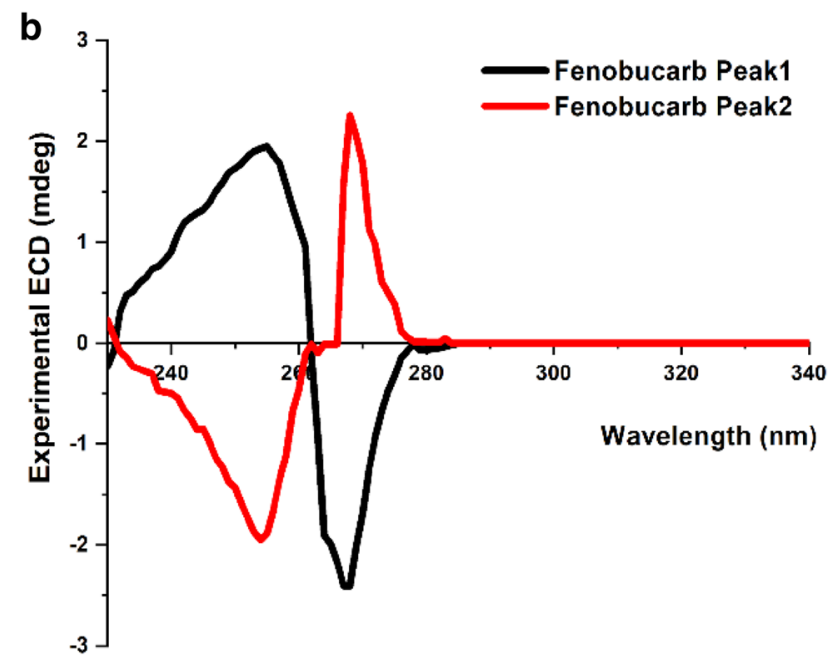

Fig. 2 The predicted and experimentally measured ECD spectra of fenobucarb enantiomers: a predicted ECD spectrogram of fenobucarb enantiomers; $\mathbf{b}$ experimentally measured ECD spectrogram 
Table 1 Effects of the mobile phase and temperature on chiral separation

\begin{tabular}{llllllll}
\hline $\begin{array}{l}\text { Acetonitrile/ } \\
\text { water } \mathrm{v} / \mathrm{v}\end{array}$ & $\begin{array}{l}\text { Temperature } \\
\left({ }^{\circ} \mathrm{C}\right)\end{array}$ & $\mathrm{t}_{1}(\mathrm{~min})$ & $\mathrm{t}_{2}(\mathrm{~min})$ & $\mathrm{k}_{1}$ & $\mathrm{k}_{2}$ & $\mathrm{a}$ & $\mathrm{Rs}$ \\
\hline $80: 20$ & 20 & 12.10 & 12.41 & 0.46 & 0.50 & 1.09 & 0.90 \\
$70: 30$ & 20 & 13.45 & 13.92 & 0.62 & 0.68 & 1.09 & 1.29 \\
$60: 40$ & 20 & 16.98 & 17.88 & 0.98 & 1.08 & 1.10 & 1.89 \\
$50: 50$ & 20 & 22.64 & 24.30 & 1.73 & 1.93 & 1.12 & 2.70 \\
$40: 60$ & 20 & 43.28 & 47.78 & 4.21 & 4.76 & 1.13 & 3.81 \\
$60: 40$ & 25 & 16.32 & 17.13 & 0.97 & 1.06 & 1.09 & 1.86 \\
$60: 40$ & 30 & 16.03 & 16.77 & 0.93 & 1.02 & 1.10 & 1.68 \\
$60: 40$ & 35 & 15.88 & 16.59 & 0.91 & 1.00 & 1.10 & 1.67 \\
$60: 40$ & 40 & 15.55 & 16.17 & 0.87 & 0.95 & 1.09 & 1.47 \\
\hline
\end{tabular}

mobile phase was acetonitrile and $0.1 \%$ formic acid water $(40: 60, v / v)$, a waste-solvent and time-consuming result should not be selected. An good resolution ( $R s=1.89)$ and a shorter retention time were obtained by using a mixture of acetonitrile and water $(60: 40, v / v)$ at a flow rate of $0.3 \mathrm{~mL} \mathrm{~min}{ }^{-1}$. The relation between temperature $\left(20^{\circ} \mathrm{C}\right.$, $25^{\circ} \mathrm{C}, 30^{\circ} \mathrm{C}, 35^{\circ} \mathrm{C}, 40^{\circ} \mathrm{C}$ ) and resolution was also referred in this paper. The Rs was decreased with the temperature rised. The Van't Hoff's equations of the two enantiomers and thermodynamic parameters were shown in Table 2. A good linearities of Van't Hoff plots were found for the enantiomers over a temperature at the range of $20-40^{\circ} \mathrm{C}$ with $R^{2}$ ranging from 0.8477 to 0.9996 . As the calculated $\Delta H, \Delta S^{0}, \Delta \Delta H^{0}$ and $\Delta \Delta S^{0}$ values shown in Table 2, the negative $\Delta H$ values present that the transfer of the enantiomers from the mobile phase to the stationary phase was driven by enthalpy; the negative $\Delta \Delta H^{0}$ and $\Delta \Delta S^{0}$ values illustrated the separation factor of the fenobucarb individuals was driven by enthalpy when the temperature below $T_{\text {iso }}$ [23]. A temperature of $20^{\circ} \mathrm{C}$ was chosen for an effective enantioselective determination. Considering an appropriate retention time and qualified resolution, acetonitrile: $0.1 \%$ formic acid water (60: 40, v/v), the column temperature $20^{\circ} \mathrm{C}$ was chosen as optimal instrumental condition.

\subsection{Optimization of sample processing}

Fruits and vegetables are a notably complex matrix consisting of chlorophyll, food colorings and lipid-soluble materials. The interfering substances always cause prominent matrix effects for analytical instruments. Furthermore, the choice of extraction solvent is a key parameter for high extraction efficiency. The extraction efficiencies from food and environmental matrices may differ from that of extracting solvent due to factors such as the hydrophilicity and hydrophobicity of pesticides. Fenobucarb has good solubility in organic solvents, such as acetonitrile and methanol. Using methanol as the extracting solvent obtained pigmented extracts and included large amounts of matrix co-extracts. Acetonitrile was selected to as an extracting solvent with high extraction efficiencies and low matrix effects. For this reason, the extraction and purification procedure was based on a QuEChERS method. [24].

\subsection{Method validation}

\subsubsection{Specificity, linearity, matrix effect stability, LOD and LOQ}

No interfering substances and target components were detected at the retention time in the control samples of four matrices. The linear calibration curves, correlation coefficient $\left(R^{2}\right)$, slope ratios, LODs and LOQs for the two enantiomers of fenobucarb in solvent and four matrices $\left(0.5-50 \mu \mathrm{g} \mathrm{L}^{-1}\right)$ were summarized in Table 3. Excellent linearities were obtained for the two individuals of fenobucarb in four matrices $\left(R^{2} \geq 0.9962\right)$. A palpable matrix suppression effect could be observed for two enantiomers of fenobucarb in cucumber, soil and rice matrices, while

Table 2 Van't Hoff equations and thermodynamic parameters

\begin{tabular}{|c|c|c|c|c|c|}
\hline Enantiomer & $\ln k^{\prime}$ & $\ln a$ & $\Delta \Delta H^{0}\left(\mathrm{~kJ} \mathrm{~mol}{ }^{-1}\right)$ & $\Delta \Delta S^{0}\left(\mathrm{~J} \mathrm{~mol}^{-1}\right)$ & $T_{\text {iso }}(\mathrm{K})$ \\
\hline (S)-(-)-fenobucarb & $\begin{aligned} \ln k_{1} & =533.8 / T-1.8329 \\
\left(R^{2}\right. & =0.9717)\end{aligned}$ & $\begin{array}{l}\ln a=51.501 / \mathrm{T}-0.0797 \\
\quad\left(R^{2}=0.8477\right)\end{array}$ & -0.428 & -0.742 & 576.8 \\
\hline (R)-(+)-fenobucarb & $\begin{aligned} \ln k_{2} & =-74,100 / T+270.44 \\
\left(R^{2}\right. & =0.9996)\end{aligned}$ & & & & \\
\hline
\end{tabular}


Table 3 Comparison of matrix-matched calibration and solvent calibration for fenobucarb enantiomers $\left(0.5-50 \mu g \mathrm{~L}^{-1}\right)$

\begin{tabular}{|c|c|c|c|c|c|c|}
\hline Enantiomer & Matrix & Regression equation & $\mathrm{R}^{2}$ & Matrix effects & $\operatorname{LOD}\left(\mu \mathrm{g} \mathrm{L}^{-1}\right)$ & LOQ $\left(\mu \mathrm{g} \mathrm{L}^{-1}\right)$ \\
\hline \multirow[t]{5}{*}{ (S)-(-)-fenobucarb } & Solvent & $y=46,416 x+5056.9$ & 0.9999 & & 0.12 & 0.40 \\
\hline & Soil & $y=24,792 x+24,728$ & 0.9991 & 0.53 & 0.08 & 0.28 \\
\hline & Cucumber & $y=16,011 x+32,559$ & 0.9975 & 0.34 & 0.16 & 0.54 \\
\hline & Rice & $y=35,519 x+24,536$ & 0.9997 & 0.76 & 0.07 & 0.28 \\
\hline & Water & $y=45,604 x+42,698$ & 0.9967 & 0.98 & 0.05 & 0.24 \\
\hline \multirow[t]{5}{*}{ (R)-(+)-fenobucarb } & Solvent & $y=47,069 x+1379.1$ & 0.9999 & & 0.12 & 0.40 \\
\hline & Soil & $y=24,217 x+3113$ & 0.9991 & 0.51 & 0.08 & 0.28 \\
\hline & Cucumber & $y=15,765 x+30,112$ & 0.9970 & 0.33 & 0.16 & 0.54 \\
\hline & Rice & $y=33,821 x+24,536$ & 0.9999 & 0.72 & 0.07 & 0.28 \\
\hline & Water & $y=46,245 x+41,402$ & 0.9962 & 0.98 & 0.05 & 0.24 \\
\hline
\end{tabular}

no significant matrix effect was observed in water. The LOD values of the two enantiomers were in the range of $0.07-0.16 \mu \mathrm{g} \mathrm{L}^{-1}$ while LOQ values were in the range of $0.24-0.40 \mu \mathrm{g} \mathrm{L}^{-1}$.

The stability of fenobucarb enantiomers in four matrices was demonstrated that no significant difference $(P>0.05)$ after storage 6 months.

\subsubsection{Accuracy and precision}

As shown in Table 4, the developed method presented sufficient recoveries and precision. The average recoveries of the (S)-(-)-fenobucarb were ranged from 81.4 to $98.6 \%$ and the intra-day RSDs $(n=5)$ and inter-day $(n=15)$ were at the ranged of $1.8-6.6 \%$ and $3.3-5.0 \%$. The average recoveries of the (R)-(+)-fenobucarb were ranged from 81.5 to $102.2 \%$, the intra- and inter-day RSDs were at the ranged of $1.8-6.6 \%$ and $3.0-5.7 \%$. The results prove that the developed method was able to provide competent accuracy and precision for fenobucarb enantiomers and can be applied stereoselective analysis in the vegetables and environmental matrices.

\subsection{Stereoselective degradation of fenobucarb in cucumber}

The degradation of fenobucarb in cucumber was shown in Fig. 3. Fenobucarb enantiomers degradation accorded well with first-order kinetics in cucumbers. The dissipation dynamics equations were: $C_{t}=0.4311 \mathrm{e}^{-0.278 t}\left(R^{2}=0.8651\right)$ for (R)-(+)-fenobucarb; $C_{t}=0.4585 \mathrm{e}^{-0.172 t}\left(R^{2}=0.8045\right)$ for (S)-(-)-fenobucarb. The half-lives were $2.49 \mathrm{~d}$ for $(\mathrm{R})$ (+)-fenobucarb and $4.03 \mathrm{~d}$ for (S)-(-)-fenobucarb with significantly different $(P=0.026<0.05$, Student's paired $t$ test). As shown in Fig. $3 \mathrm{~b}$, the EF value was nearly 0.5 at the beginning, which proved the fenobucarb exist in cucumber as a racemate. Nevertheless, EF values reached 0.2 at $10 \mathrm{~d}$, which indicated that most of fenobucarb exist as the form of (S)-(-)-fenobucarb. It means the (R)-(+)fenobucarb was preferentially degraded comparing to (S)-(-)-fenobucarb in cucumber. A reasonable exposition is that when fenobucarb was applied in the cucumbers, stereoselective metabolism in plants was involved the stereoselective process caused by corresponding plant functional enzymes. In addition, numerous researches suggest that plant enzyme system takes effect in the stereoselective degradation [25].

In general, the degradation of fenobucarb in cucumber was enantioselective, (R)-(+)-fenobucarb degraded faster than (S)-(-)-fenobucarb in cucumbers.

\section{Conclusion}

In this study, the absolute configuration of fenobucarb enantiomers were confirmed by comparing the experimental ECD spectrum and predicted ECD. A baseline separation between the $\mathrm{R}$ - and $\mathrm{S}$-enantiomers was achieved at optimized condition. A novel and dependable enantioselective method for the coinstantaneous quantitative determination of enantiomers in four kinds of matrices has been successfully developed and validated. (R)-(+)fenobucarb degraded faster than (S)-(-)-fenobucarb in cucumbers. The developed method may be useful for determining trace quantities of fenobucarb enantiomers in food and environmental samples and could be applied for stereoselective degradation studies in plant and environmental samples. It provided additional information for reliable risk assessment of fenobucarb. 
Table 4 Accuracy and precision of the proposed method in four matrices

\begin{tabular}{|c|c|c|c|c|c|c|c|c|c|}
\hline \multirow[t]{3}{*}{ Compound } & \multirow[t]{3}{*}{ Matrix } & \multirow{3}{*}{$\begin{array}{l}\text { Spiked level } \\
\left(\mu \mathrm{g} \mathrm{mL}^{-1}\right)\end{array}$} & \multicolumn{6}{|c|}{ Intra-day $(n=5)$} & \multirow{3}{*}{$\begin{array}{l}\text { Inter-day }(n=15) \\
\text { RSD (\%) }\end{array}$} \\
\hline & & & \multicolumn{2}{|l|}{ Day 1} & \multicolumn{2}{|l|}{ Day 2} & \multicolumn{2}{|l|}{ Day 3} & \\
\hline & & & $\begin{array}{l}\text { Average } \\
\text { recoveries } \\
(\%)\end{array}$ & RSD (\%) & $\begin{array}{l}\text { Average } \\
\text { recoveries } \\
(\%)\end{array}$ & RSD (\%) & $\begin{array}{l}\text { Average } \\
\text { recoveries } \\
(\%)\end{array}$ & RSD (\%) & \\
\hline \multirow[t]{12}{*}{ (S)-(-)-fenobucarb } & \multirow[t]{3}{*}{ Soil } & 0.005 & 95.3 & 5.6 & 96.5 & 3.6 & 97.6 & 4.3 & 4.8 \\
\hline & & 0.05 & 83.7 & 3.4 & 84.5 & 3.2 & 83.9 & 4.4 & 3.9 \\
\hline & & 0.5 & 81.4 & 4.0 & 82.6 & 2.7 & 83.5 & 3.5 & 3.7 \\
\hline & \multirow[t]{3}{*}{ Cucumber } & 0.005 & 97.8 & 6.4 & 93.1 & 4.8 & 98.6 & 5.4 & 5.8 \\
\hline & & 0.05 & 90.2 & 3.3 & 93.0 & 5.3 & 94.1 & 3.9 & 4.3 \\
\hline & & 0.5 & 96.4 & 1.8 & 89.9 & 4.1 & 93.2 & 3.6 & 4.0 \\
\hline & \multirow[t]{3}{*}{ Rice } & 0.005 & 94.0 & 6.2 & 91.2 & 5.0 & 92.2 & 4.4 & 5.0 \\
\hline & & 0.05 & 85.8 & 4.5 & 85.0 & 3.8 & 87.1 & 4.2 & 4.0 \\
\hline & & 0.5 & 80.4 & 3.5 & 81.7 & 5.3 & 83.1 & 6.4 & 5.1 \\
\hline & \multirow[t]{3}{*}{ Water } & 0.005 & 97.9 & 3.6 & 98.6 & 4.1 & 98.2 & 2.8 & 3.3 \\
\hline & & 0.05 & 93.0 & 4.1 & 94.1 & 2.5 & 95.4 & 3.3 & 3.3 \\
\hline & & 0.5 & 93.2 & 4.1 & 92.9 & 4.1 & 88.7 & 3.4 & 4.3 \\
\hline \multirow[t]{12}{*}{ (R)-(+)-fenobucarb } & \multirow[t]{3}{*}{ Soil } & 0.005 & 95.4 & 4.7 & 95.8 & 4.2 & 97.3 & 5.0 & 4.4 \\
\hline & & 0.05 & 83.6 & 4.6 & 82.0 & 3.9 & 81.9 & 4.4 & 4.1 \\
\hline & & 0.5 & 81.5 & 4.3 & 82.9 & 3.6 & 84.3 & 4.2 & 4.0 \\
\hline & \multirow[t]{3}{*}{ Cucumber } & 0.005 & 95.8 & 4.6 & 91.4 & 3.1 & 98.8 & 3.3 & 4.8 \\
\hline & & 0.05 & 90.3 & 4.9 & 92.2 & 5.1 & 93.0 & 3.8 & 4.5 \\
\hline & & 0.5 & 92.6 & 2.3 & 88.9 & 2.4 & 91.8 & 4.0 & 3.3 \\
\hline & \multirow[t]{3}{*}{ Rice } & 0.005 & 93.5 & 5.0 & 91.5 & 4.3 & 94.7 & 6.0 & 5.0 \\
\hline & & 0.05 & 87.0 & 3.8 & 86.6 & 4.4 & 84.3 & 6.1 & 4.7 \\
\hline & & 0.5 & 83.4 & 6.4 & 83.3 & 6.6 & 83.7 & 5.5 & 5.7 \\
\hline & \multirow[t]{3}{*}{ Water } & 0.005 & 102.2 & 2.8 & 102.0 & 4.0 & 101.0 & 3.0 & 3.0 \\
\hline & & 0.05 & 94.0 & 3.6 & 95.8 & 3.3 & 95.4 & 2.6 & 3.1 \\
\hline & & 0.5 & 94.8 & 3.7 & 96.6 & 3.3 & 90.6 & 3.0 & 4.2 \\
\hline
\end{tabular}
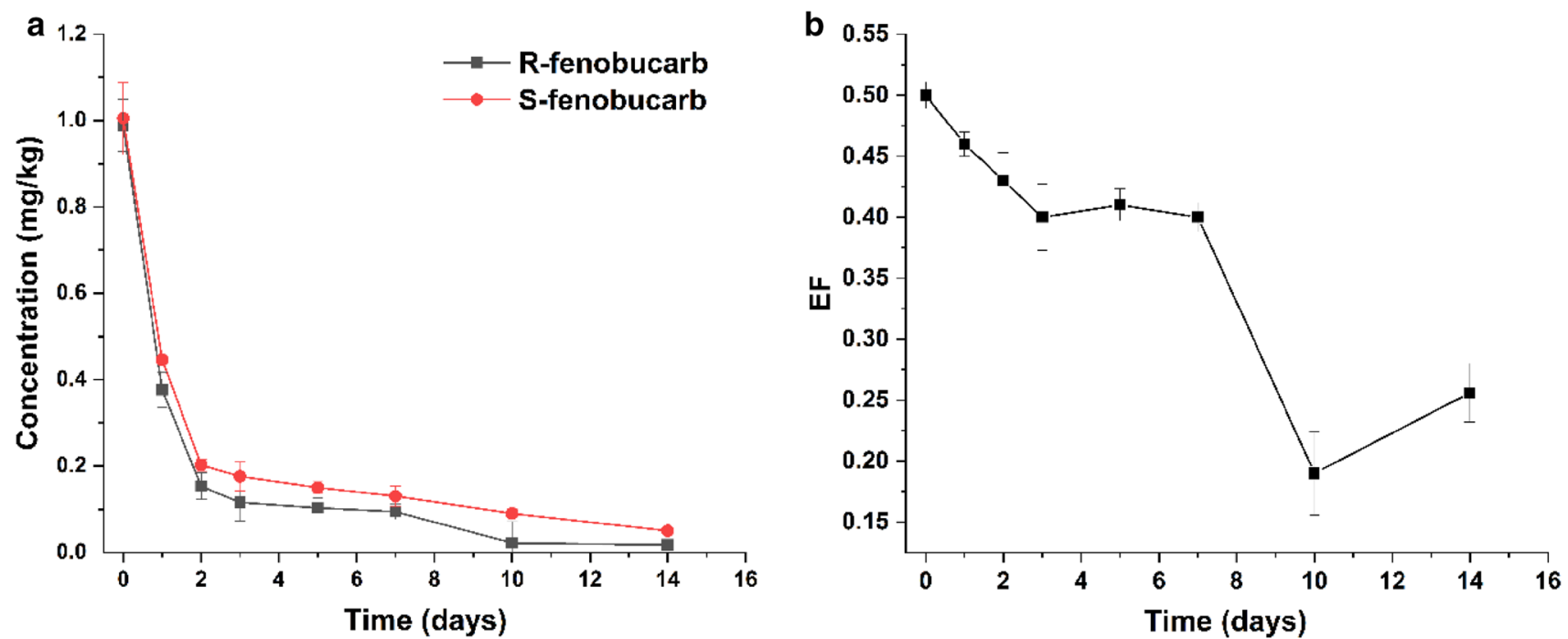

Fig. 3 The degradation of the fenobucarb enantiomers in cucumbers (a) and EF value variation of fenobucarb enantiomers (b) 
Acknowledgements This study was supported by the National Key Research and Development Program of China (2016YFD0200207). We are grateful to Hu Zhang (Zhejiang Academy of Agriculture Sciences) for calculating the absolute configuration of fenobucarb enantiomers using Gaussian 09 software.

\section{Compliance with ethical standards}

Conflict of interest The authors declare that they have no conflict of interest.

\section{References}

1. Fukuto TR (1990) Mechanism of action of organophosphorus and carbamate insecticides. Environ Health Perspect 87:245-254

2. Kim I, Kim DU, Kim NH, Ka JO (2004) Isolation and characterization of fenobucarb-degrading bacteria from rice paddy soils. Biodegradation 25:383-394

3. Fahmy MA, Fukuto TR, Myers RO, March RB (1970) Selective toxicity of new N-phosphorothioylcarbamate esters. J Agric Food Chem 18:793-796

4. Sastry KV, Siddiqui AA (1982) Chronic toxic effects of the carbamate pesticide sevin on carbohydrate metabolism in a freshwater snakehead fish, Channa punctatus. Toxicol Lett 14:123-130

5. Chapalamadugu S, Chaudhry GR (1992) Microbiological and biotechnological aspects metabolism of carbamates and organophosphates. Crit Rev Biotechnol 12:357-389

6. Zheng WJ, Park JA, Zhang D, Abd El-Aty AM, Kim SK, Cho SH, Choi JM, Shim JH, Chang BJ, Kim JS, Shin HC (2017) Determination of fenobucarb residues in animal and aquatic food products using liquid chromatography-tandem mass spectrometry coupled with a QuEChERS extraction method. J Chromatogr B 1058:1-7

7. Liang WQ, Wang JT, Zang XH, Dong WH, Wang C, Wang Z (2017) Barley husk carbon as the fiber coating for the solid-phase microextraction of twelve pesticides in vegetables prior to gas chromatography-mass spectrometric detection. J Chromatogr A 1491:9-15

8. Qi P, Wang X, Zhang H, Wang X, Xu H, Wang Q (2015) Rapid enantioseparation and determination of isocarbophos enantiomers in orange pulp, peel, and kumquat by chiral HPLC-MS/MS. J Chromatogr B 8:531-538

9. Lee SR, Park HB, Kim KH (2018) Highly sensitive, simple, and costand time-effective method to determine the absolute configuration of a secondary alcohol using competing enantioselective acylation coupled with LC/MS. Anal Chem 90:13212-13216

10. Zhang Q, Gao BB, Tian MM, Shi HY, Wang MH (2016) Enantioseparation and determination of triticonazole enantiomers in fruits, vegetables, and soil using efficient extraction and cleanup methods. J Chromatogr B 1009:130-137

11. Garrison AW (2006) Probing the enantioselectivity of chiral pesticides. Environ Sci Technol 40:16-23

12. Sun DL, Qiu J, Wu YJ, Liang HW, Liu CL, Li L (2012) Enantioselective degradation of indoxacarb in cabbage and soil under field conditions. Chirality 24:628-633
13. Wang H, Dong F, Li Y, Chen X, Cheng Y, Xiang W, Zheng Y (2013) Enantioselective determination of the insecticide indoxacarb in cucumber and tomato by chiral liquid chromatography-tandem mass spectrometry. Chirality 25:350-354

14. Ding S, Kolbanovskiy A, Durandin A, Crean C, Shafirovich V, Broyde S, Geacintov NE (2009) Absolute configurations of DNA lesions determined by comparisons of experimental ECD and ORD spectra with DFT calculations. Chirality 21:E231-E241

15. Ding S, Jia L, Durandin A, Crean C, Kolbanovskiy A, Shafirovich V, Broyde S, Geacintov NE (2009) Absolute configurations of spiroiminodihydantoin and allantoin stereoisomers: comparison of computed and measured electronic circular dichroism spectra. Chem Res Toxicol 22:1189-1193

16. O'Brien T, Crocker L, Thompson R, Thompson K, Toma PH, Conlon DA, Feibush B, Moeder C, Bicker G, Grinberg N (1997) Mechanistic aspects of chiral discrimination on modified cellulose. Anal Chem 69:1999-2007

17. Zhang Q, Tian MM, Wang MY, Shi HY, Wang MH (2014) Simultaneous enantioselective determination of triazole fungicide flutriafol in vegetables, fruits, wheat, soil, and water by reversed-phase high-performance liquid chromatography. J Agric Food Chem 62:2809-2815

18. Adhikari S, Paik MJ, Lee W (2018) Liquid chromatographic enantiomeric separation of chiral aliphatic amines using 2-hydroxynaphthaldehyde as a derivatizing agent on polysaccharide-derived chiral stationary phases. Chromatographia 81:1337-1344

19. Vashistha VK, Marten J, Bhushan R (2017) Sensitive RP-HPLC enantioseparation of (RS)-ketamine via chiral derivatization based on (S)-levofloxacin. Chromatographia 80:1501-1508

20. Gao BB, Zhang Q, Tian MM, Zhang ZX, Wang MH (2016) Enantioselective determination of the chiral pesticide isofenphosmethyl in vegetables, fruits, and soil and its enantioselective degradation in pak choi using HPLC with UV detection. Anal Bioanal Chem 408:6719-6727

21. Ulrich EM, Helsel DR, Foreman WT (2003) Complications with using ratios for environmental data: comparing enantiomeric ratios (ERs) and enantiomer fractions (EFs). Chemosphere 53:531-538

22. Péter A, Vékes $E$, Armstrong DW (2002) Effects of temperature on retention of chiral compounds on a ristocetin A chiral stationary phase. J Chromatogr A 958:89-107

23. Moskal'ová $M$, Kozlov $O$, Gondová T, Budovská $M$, Armstrong DW (2017) HPLC enantioseparation of novel spirobrassinin analogs on the cyclofructan chiral stationary phases. Chromatographia 80:52-63

24. Wilkowska A, Biziuk M (2011) Determination of pesticide residues in food matrices using the QuEChERS methodology. Food Chem 125:803-812

25. Fu SD, Liu XG, Zheng YQ, Cao Q, Li CJ (2010) Stereoselective degradation of fungicide triadimenol in cucumber plants. Chirality 22:292-298

Publisher's Note Springer Nature remains neutral with regard to jurisdictional claims in published maps and institutional affiliations. 